\title{
Dynamical equilibrium between excitons and trions in CdTe quantum wells in high magnetic fields
}

\author{
C. R. L. P. N. Jeukens, P. C. M. Christianen, and J. C. Maan \\ Research Institute for Materials, High Field Magnet Laboratory, University of Nijmegen, Toernooiveld 1, \\ 6525 ED Nijmegen, The Netherlands \\ D. R. Yakovlev and W. Ossau \\ Physikalisches Institut der Universität Würzburg, Am Hubland, 97074 Würzburg, Germany \\ V. P. Kochereshko \\ A. F. Ioffe, Physico-Technical Institute, Russian Academy of Sciences, 194021 St. Petersburg, Russia \\ T. Wojtowicz, G. Karczewski, and J. Kossut \\ Institute of Physics, Polish Academy of Sciences, Al. Lotnikow, 32/46, 02-668 Warsaw, Poland
}

(Received 12 July 2002; published 30 December 2002)

\begin{abstract}
The formation of two-dimensional negatively charged excitons (negative trions) out of excitons and free electrons is found to be determined by a dynamical equilibrium. This dynamical equilibrium consists of a chemical equilibrium, relating the trion, exciton, and electron populations, modified by finite formation and recombination times of (charged) excitons, as is evidenced by a magnetic-field-dependent photoluminescence (PL) and far-infrared study of doped $\mathrm{CdTe} / \mathrm{CdMgTe}$ quantum wells. The data show that the trion formation is entirely driven by the occupation of the spin-split trion, exciton and electron levels. Incorporation of the proposed trion formation scheme into a rate equation model gives a proper description of the experimental data, leading to values of the formation, recombination, and spin-flip times of trions and excitons that are in good agreement with results of time-resolved experiments in the literature. The model elucidates the effect of the heavy-hole splitting on the polarization degree of the trion PL in a magnetic field and the influence of the dark excitons on the trion formation.
\end{abstract}

DOI: 10.1103/PhysRevB.66.235318

PACS number(s): 78.55.Et, 71.35.-y, 78.20.Ls

\section{INTRODUCTION}

The optical spectrum of undoped semiconductor quantum-well (QW) structures is dominated by excitonic transitions. In the case of a low excess density of electrons $e$ (or holes $h$ ), a photoexcited exciton $(X)$ can capture an extra electron (or hole) to form a negatively $\left(X^{-}\right)$(or positively, $X^{+}$) charged exciton, or so-called trion. Both $X^{-}$(two electrons bound to one hole) and $X^{+}$(one electron bound to two holes) lead to an additional peak in the optical spectrum at a slightly lower energy than $X$. Although Lambert already predicted the existence of charged excitons in the 1950's, ${ }^{1}$ negative trions were only recently identified experimentally by Kheng et al., ${ }^{2}$ utilizing the distinct polarization properties of the trion absorption peak in a magnetic field.

Since this first experimental identification in CdTe-based QW's, negative and positive trions have been observed in other materials as well, such as GaAs- (Refs. 3-5) and ZnSe-based ${ }^{6}$ heterostructures. Numerous properties have been investigated, such as the binding energy of singlet and triplet trion states with $^{7-13}$ and without ${ }^{14,15}$ a magnetic field, the optical spectrum of trions with varying excess carrier density, ${ }^{16}$ combined exciton-electron cyclotron resonances, ${ }^{17}$ trion localization, ${ }^{18-21}$ and trion dynamics on a picosecond time scale. ${ }^{5,22-25}$ Time-resolved experiments have shown that the characteristic times (formation times of trions, recombination times of excitons and trions, and spin-flip times) are of the same order of magnitude, irrespective of the material studied. ${ }^{5,23}$ As a consequence, it seems reasonable to con- clude that in cw experiments a thermodynamic equilibrium between excitons, trions, and electrons is also not established. That is, when the trion recombination time is comparable to the trion formation time, a considerable amount of excitons recombines before forming a trion. The relative intensity of the trion and exciton emission is therefore expected to depend on the ratio between the recombination and formation times, which is often ignored in the analysis of cw experiments. ${ }^{26-29}$ In previous studies the trion formation process was described as a chemical equilibrium between the populations of the excitons, trions, and free electrons, independent of the relevant time constants of the system. Furthermore, the contribution of the dark, i.e., nonradiative, exciton states $^{23,30}$ is often neglected, ${ }^{31-34}$ because their direct effect is not evident. Although these states do not appear in the optical spectrum, their contribution to the trion formation process is expected to be equal to that of the bright, radiative exciton states.

In a magnetic field the trion, exciton, and electron energy levels split due to the Zeeman effect. This fact has helped in the identification of trions, since the polarization of the trion absorption was found to follow the known field-dependent electron polarization, as opposed to the regular exciton peak, which is only weakly polarized. ${ }^{2,3}$ In magnetophotoluminescence (MPL) measurements the polarization behavior is more complicated to understand, since it also depends on the occupancies of all relevant energy levels, determined by the relative time constants and the (field-induced) splittings of the energy levels in the system. As a consequence, pro- 
nounced MPL polarization effects have been observed, and various different explanations have been put forward. In fact, as we will show here, monitoring the polarization of the exciton and trion emission in a magnetic field enables the determination of the relative importance for the trion formation of the factors mentioned above. For instance, the spinsplitting of the singlet trion depends solely on the heavy-hole splitting. By measuring the trion polarization, the role of the heavy-hole splitting can therefore be determined. Furthermore, in a magnetic field the contribution of dark excitons to the trion formation can become significant when a dark exciton state becomes lowest in energy, leading to a large population. By using far-infrared (FIR) radiation that induces changes in the occupancies of the dark and bright exciton states leading to changes in the photoluminescence (PL) emission, the role of the dark exciton states can be made visible.

In this paper we report a comprehensive study of the negatively charged exciton formation process in $\mathrm{CdTe} /$ CdMgTe QW's using cw PL spectroscopy and FIR-based optically detected resonance (ODR) (Refs. 35 and 36) spectroscopy in high magnetic fields. We show that the formation can be described by a dynamical equilibrium, consisting of a chemical equilibrium, modified by finite recombination times and spin-flip times of the excitons and trions. This description resolves the apparent discrepancy between the cw and time-resolved experiments. Moreover, we show that ODR data reveal the importance of the dark, nonradiative exciton states in the trion formation and that the polarization of the trion emission depends on the splitting of the heavy-hole energy levels. We present model calculations that explain the polarization of the trion observed in the magneto-PL data and the ODR data. The values for all relevant time constants are found to be in good agreement with time-resolved experiments, showing that one can develop a consistent and comprehensive physical picture describing both $\mathrm{cw}$ and timeresolved experiments.

The paper is organized as follows: after discussing the experimental details in Sec. II, the experimental results are presented in Sec. III. In Sec. IV we analyze the data using a rate equation model that includes the formation scheme proposed in Sec. III. We end with the conclusions in Sec. V.

\section{EXPERIMENTAL DETAILS}

For our studies we used two 8-nm-wide modulationdoped $\mathrm{CdTe} / \mathrm{Cd}_{0.7} \mathrm{Mg}_{0.3} \mathrm{Te}$ single QW structures grown by molecular-beam epitaxy on (100)-oriented GaAs substrates. CdTe exhibits strong excitonic effects, yielding an exciton binding energy of about $10 \mathrm{meV}$ in bulk CdTe. Confinement in the QW increases this value up to about $20 \mathrm{meV}$ resulting in a large trion binding energy of $E_{b} \sim 3 \mathrm{meV},{ }^{2}$ as compared to $\sim 1 \mathrm{meV}$ for GaAs QW's. ${ }^{3,5}$ The PL spectra only reveal singlet trion states (anti-parallel electron spins) and no triplet trion states (parallel electron spins) in a magnetic field, which simplifies our discussion. The two samples originate from the same wafer grown by a wedged doping technique using Iodine as the dopant ${ }^{37}$ and differ only in their background electron density. The density of the first sample (sample I) was found to be $n_{e}=2 \times 10^{10} \mathrm{~cm}^{-2}$, by measuring the polarization degree of the trion peak in the reflection spectra as a function of the magnetic field. ${ }^{38,39}$ In this sample the density of electrons was always larger than the density of trions $\left(n_{X^{-}}\right)$and excitons $\left(n_{X}\right)$ (i.e., $\left.n_{e} \gg n_{\mathrm{X}^{-}}+n_{\mathrm{X}}\right)$ for the optical excitation conditions used in the experiments. Therefore, there was no restriction in trion formation due to a shortage of electrons. The second sample (sample II) was nominally undoped, but under optical excitation there was a high enough electron density to observe trion emission besides the regular exciton emission.

For the PL experiments we used a HeNe laser (nonresonant excitation). Fiber optics was used to guide the laser beam toward the sample and to collect the PL. After passing through a monochromator, the PL was detected by a CCD camera. For the PL excitation (PLE) measurements the monochromator was set at a fixed detection energy on the low-energy side of the trion PL peak, while scanning the wavelength of a Ti:sapphire laser. In this case the PL emission was collected by a cooled GaAs photomultiplier tube. For the measurements in the magnetic field we used a setup which allowed us to perform PL and ODR measurements simultaneously. ${ }^{35,36}$ The sample was placed in the Faraday geometry in a cryostat at a temperature $T=1.4 \mathrm{~K}$ inside a Bitter magnet, which can produce dc fields up to $17 \mathrm{~T}$. To perform polarized measurements a $\lambda / 4$ plate and a polarizer were mounted directly above the sample in the cryostat before the luminescence entered the fiber. The FIR radiation was produced by a methanol-gas-based FIR laser system, which was pumped by a $40-\mathrm{W} \mathrm{cw} \mathrm{CO}_{2}$ laser. The FIR laser lines used in the experiment were $\lambda_{\mathrm{FIR}}=118.8 \mu \mathrm{m}(P$ $\simeq 10 \mathrm{~mW}), \quad \lambda_{\mathrm{FIR}}=570 \mu \mathrm{m} \quad(P \simeq 3 \mathrm{~mW}), \quad$ and $\quad \lambda_{\mathrm{FIR}}$ $=96.5 \mu \mathrm{m}(P \simeq 2 \mathrm{~mW})$. The FIR radiation was guided by oversized waveguide tubes to the backside of the sample, so that the sample could be simultaneously irradiated by the $\mathrm{HeNe}$ and FIR laser light. In order to record the ODR signal, i.e., the changes in PL due to the absorption of FIR radiation, the FIR beam was mechanically chopped, while the PL was monitored using a cooled GaAs photomultiplier tube and a lock-in amplifier at the frequency of the chopper. When sweeping the magnetic field, the monochromator was set to follow the PL peak position of the trion or exciton emission, which shifts in a magnetic field. These measurements resulted in four curves (trions and excitons for both $\sigma^{-}$and $\sigma^{+}$polarizations) as a function of the magnetic field.

\section{EXPERIMENTAL RESULTS}

A typical PL spectrum of sample I $\left(n_{e}=2 \times 10^{10} \mathrm{~cm}^{-2}\right)$ at $B=0 \mathrm{~T}$ and $T=1.4 \mathrm{~K}$ is shown in Fig. 1(a). The spectrum is dominated by a trion emission peak which has a slightly lower energy than a less intense exciton peak. The energy splitting $(3.9 \mathrm{meV})$ between the peaks is roughly given by the sum of the binding energy of the second electron of $X^{-}$ $\left(E_{b} \sim 3.1 \mathrm{meV}\right)$ plus the energy needed to set free the second electron into the lowest unoccupied state at the Fermi-energy. ${ }^{16}$ The PLE [dotted line in Fig. 1(a)] and the reflection (not shown) spectra also consist of two peaks, with a Stokes shift of $0.6 \mathrm{meV}$ with respect to the PL peaks. The presence of the $X^{-}$resonance in PLE and reflection, al- 


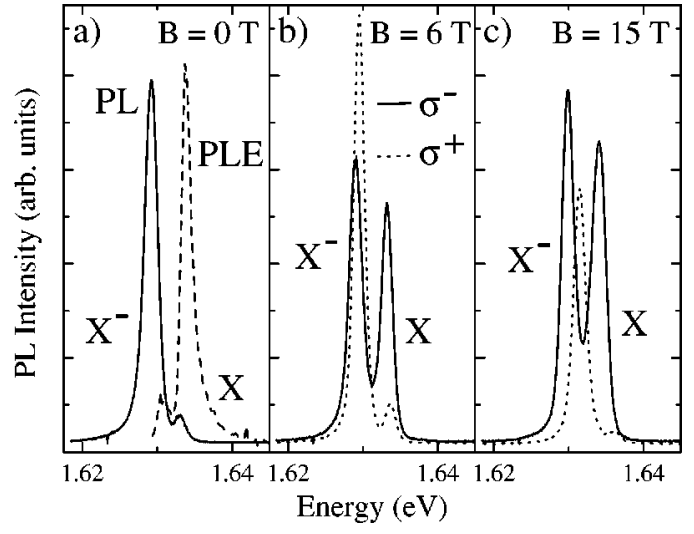

FIG. 1. (a) Typical PL spectrum (solid line) at $B=0 \mathrm{~T}$ and $T$ $=1.4 \mathrm{~K}$ of the 8 -nm-thick CdTe/ $\mathrm{Cd}_{0.7} \mathrm{Mg}_{0.3} \mathrm{Te}$ single $\mathrm{QW}$ with $n_{e}$ $=2 \times 10^{10} \mathrm{~cm}^{-2}$ (sample I), which shows the trion $\left(X^{-}\right)$and exciton $(X)$ emission peaks. The dotted line represents the PLE spectrum (detection energy $1.6286 \mathrm{eV}$ ). PL spectra at (b) $B=6 \mathrm{~T}$, and (c) $B=15 \mathrm{~T}$ showing large changes in trion and exciton emission intensity. The solid spectra represent the $\sigma^{-}$polarized emission, the dotted spectra the $\sigma^{+}$polarized emission.

though weak, confirms its intrinsic origin and other explanations such as impurity related transitions can be ruled out. The relative intensities of the $X$ and $X^{-}$resonances in the PLE spectrum indicate that in this sample the oscillator strength of the exciton is larger than that of the trion. In contrast, the PL emission strongly depends on the occupation of the levels as well. The intense $X^{-}$peak corresponds, therefore, to a high trion density, and implies that the trion formation out of excitons and free electrons is very efficient.

In $\mathrm{cw}$ studies the trion formation is frequently described in terms of a chemical equilibrium: ${ }^{26-29}$

$$
X+e \rightleftharpoons X^{-} .
$$

The densities of trions and excitons are then related according to the well-known law of mass action, ${ }^{40}$

$$
\frac{n_{X} n_{e}}{n_{X^{-}}}=\frac{k_{B} T m_{e}}{2 \pi \hbar^{2}} \frac{m_{X}}{m_{X^{-}}} \exp \left[-E_{b} / k_{B} T\right],
$$

where $m_{i}$ denotes the effective mass of electrons $\left(m_{e}\right.$ $\left.=0.11 m_{0}\right)$, excitons $\left(m_{X}=0.59 m_{0}\right)$, and trions $\left(m_{X^{-}}\right.$ $\left.=0.70 m_{0}\right)$, in which $m_{0}$ is the electron rest mass. When $n_{X}, n_{X^{-}} \ll n_{e}$, which is our experimental case, this law implies that at a fixed temperature the ratio $n_{X} / n_{X^{-}}$is given by $n_{e}$ and the fixed parameters $m_{i}$ and $E_{b}$. With increasing $n_{e}$ the number of trions increases, as is expected and which is experimentally observed. ${ }^{28,29,35}$ For $n_{e}=2 \times 10^{10} \mathrm{~cm}^{-2}$ (sample I) and at low temperatures $(T<5 \mathrm{~K})$ this equation predicts a dominating trion population, while the exciton population is virtually zero. Our observation of a nonzero exciton PL peak clearly shows the presence of an exciton population $^{41}$ [see Fig. 1(a)], indicating that this system does not reach a state of chemical equilibrium nor a state of thermal equilibrium for which the same arguments hold. The observation of exciton emission suggests that the trion formation rate is in direct competition with the exciton recom-

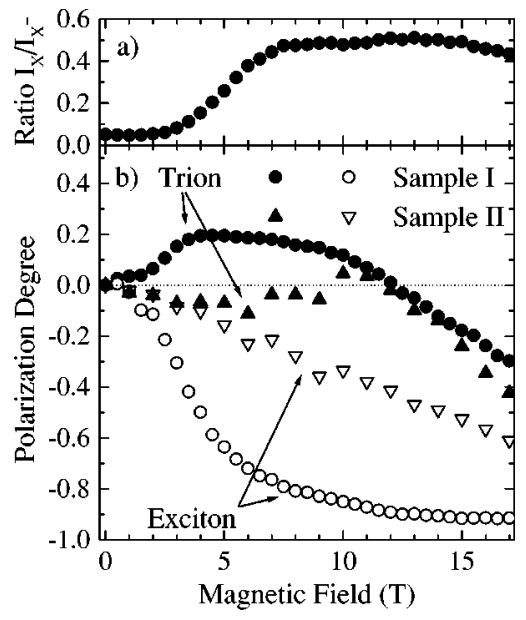

FIG. 2. (a) The intensity ratio $\left(I_{X}^{\sigma^{-}}+I_{X}^{\sigma^{+}}\right) /\left(I_{X^{-}}^{\sigma^{-}}+I_{X^{-}}^{\sigma^{+}}\right)$, which increases in a magnetic field. (b) The polarization degree $\mathcal{P}$ $=\left(I^{\sigma^{+}}-I^{\sigma^{-}}\right) /\left(I^{\sigma^{+}}+I^{\sigma^{-}}\right)$of the trions (solid symbols) and excitons (open symbols) at $T=1.4 \mathrm{~K}$ for sample I with $n_{e}=2 \times 10^{10} \mathrm{~cm}^{-2}$ (circles) as a function of the magnetic field showing a change of sign in the trion polarization at $B=12 \mathrm{~T}$. The polarization degree of sample II (nominally undoped, triangles) is also plotted, showing the large influence of the electron concentration on the formation process.

bination rate as a consequence of the fact that the characteristic times are of the same order of magnitude, ${ }^{5,23}$ i.e., that part of the excitons recombine before forming a trion.

\section{A. PL measurements in magnetic fields}

When a magnetic field is applied to the system the energy levels of the electrons, excitons and trions split due to the Zeeman effect, leading to large changes in the trion and exciton PL intensities (Fig. 1). 6 ,42,31,32 The PL spectra show two main effects [compare Figs. 1(a), 1(b), and 1(c) at $B=0,6$, and $15 \mathrm{~T}]$ : (1) For low magnetic fields $(B=6 \mathrm{~T})$ the $\sigma^{+}$-polarized trion emission intensity $\left(I_{X^{-}}^{\sigma^{+}}\right)$is stronger than the $\sigma^{-}$-polarized trion emission intensity $\left(I_{X^{-}}^{\sigma^{-}}\right)$, while at high magnetic fields $(B=15 \mathrm{~T})$ the situation is reversed. The exciton emission becomes strongly $\sigma^{-}$polarized in a magnetic field $\left(I_{X}^{\sigma^{-}}>I_{X}^{\sigma^{+}}\right)$. A similar trion polarization is also observed in a similar CdTe sample ${ }^{31}$ containing a background electron density of $n_{e}=8 \times 10^{10} \mathrm{~cm}^{-2}$ and has also been reported for modulation doped $\mathrm{CdTe} / \mathrm{CdZnTe}$ quantum wells. $^{42}$ (2) The total exciton emission intensity $\left(I_{X}=I_{X}^{\sigma^{-}}\right.$ $+I_{X}^{\sigma^{+}}$) increases in a magnetic field at the expense of the trion emission $\left(I_{X^{-}}=I_{X^{-}}^{\sigma^{-}}+I_{X^{-}}^{\sigma^{+}}\right)$, as can be readily seen in Fig. 2(a), which displays $I_{X} / I_{X^{-}}$, indicating that the magnetic field reduces the efficiency of trion formation.

The polarization behavior of the trions and excitons is clearly seen by plotting the polarization degree $\mathcal{P}=\left(I^{\sigma^{+}}\right.$ $\left.-I^{\sigma^{-}}\right) /\left(I^{\sigma^{+}}+I^{\sigma^{-}}\right)$[Fig. 2(b)]. The trion $\mathcal{P}$ is positive at low fields and changes sign at $B=12 \mathrm{~T}$, while the exciton $\mathcal{P}$ becomes increasingly negative with increasing magnetic field. To show the strong influence of the electron concentra- 


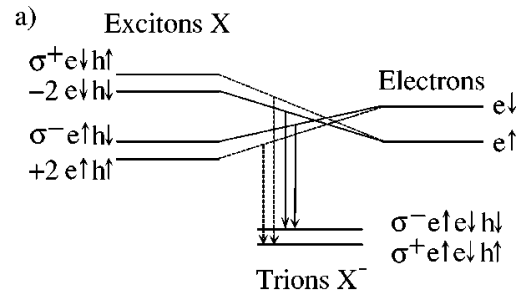

b)

$$
\begin{aligned}
& \mathrm{X}_{\sigma^{+}}+\mathrm{e} \uparrow \leftrightharpoons \mathrm{X}_{\sigma^{+}}^{-} \\
& \mathrm{x}_{-2}+\mathrm{e} \uparrow \leftrightharpoons \mathrm{X}_{\sigma^{-}} \\
& \downarrow \\
& \mathrm{X}_{\sigma^{-}}+\mathrm{e} \downarrow \leftrightharpoons \mathrm{X}_{\sigma^{-}}^{-} \\
& \mathrm{X}_{+2}+\mathrm{e} \downarrow \leftrightharpoons \mathrm{X}_{\sigma^{+}}
\end{aligned}
$$

FIG. 3. (a) Energy-level diagram for the $\mathrm{CdTe} / \mathrm{Cd}_{0.7} \mathrm{Mg}_{0.3} \mathrm{Te}$ QW, showing the spin-split levels of the trions, excitons, and electrons in moderate magnetic fields $(2<B<10 \mathrm{~T})$. The arrows denote the combinations of excitons and electrons that can form a (singlet) trion. (b) The excitons and electrons become oppositely polarized via thermalization over the Zeeman levels in a magnetic field (vertical arrows) resulting in a reduced formation rate of trions (for both $X_{\sigma^{-}}^{-}$and $X_{\sigma^{+}}^{-}$).

tion on the formation process, we have also plotted $\mathcal{P}$ of sample II in Fig. 2(b) (triangles). The positive $\mathcal{P}$ for the trion PL is absent for this sample, while the exciton PL is still negatively polarized although much less pronounced.

To explain the observed PL emission, the energy levels of the exciton and trion states and the allowed (singlet) trion formation processes need to be considered (Fig. 3). The spin states of the electrons $(e)$ and holes $(h)$ that build up the excitons and trions are denoted by arrows: $\uparrow$ for spin up and $\downarrow$ for spin down. Besides the exciton and trion levels which appear in the PL spectrum, the diagram shows the dark, nonradiative excitons $\left(X_{+2}\right.$ and $\left.X_{-2}\right)$, which do not appear in the spectrum but are expected to contribute to the trion formation. The exciton splitting is determined by the electron as well as the heavy-hole splitting. In contrast, the trion splitting depends only on the heavy-hole splitting, since both electron spins are present in a singlet trion. Unfortunately, the trion splitting cannot be directly deduced from the PL spectrum, because the electron splitting also contributes to the energy difference of the PL peaks [see Fig. 4(a)], as the two-dimensional electron-gas (2DEG) electron is the final state after recombination of $X^{-}$. However, since the electron splitting in our samples is accurately measured $\left[g_{e}=-1.46\right.$ (Ref. 43)], the heavy-hole splitting can be deduced by subtracting the electron splitting [dashed line in Fig. 4(b)] from the measured trion splitting. The open symbols in Fig. 4(b) show the resulting heavy-hole splitting. Note that the hole spin splitting is very small and negative for low fields $(B<10 \mathrm{~T})$ and changes sign with increasing field. This procedure enables the construction of the complete scheme of all field dependent energy levels displayed in Fig. 3 (see Sec. IV A).

The observed increase of the exciton emission relative to the trion emission in a magnetic field [see Fig. 2(a)] is qualitatively explained by considering the effect of the polarization of the electrons and excitons in a magnetic field [Fig.

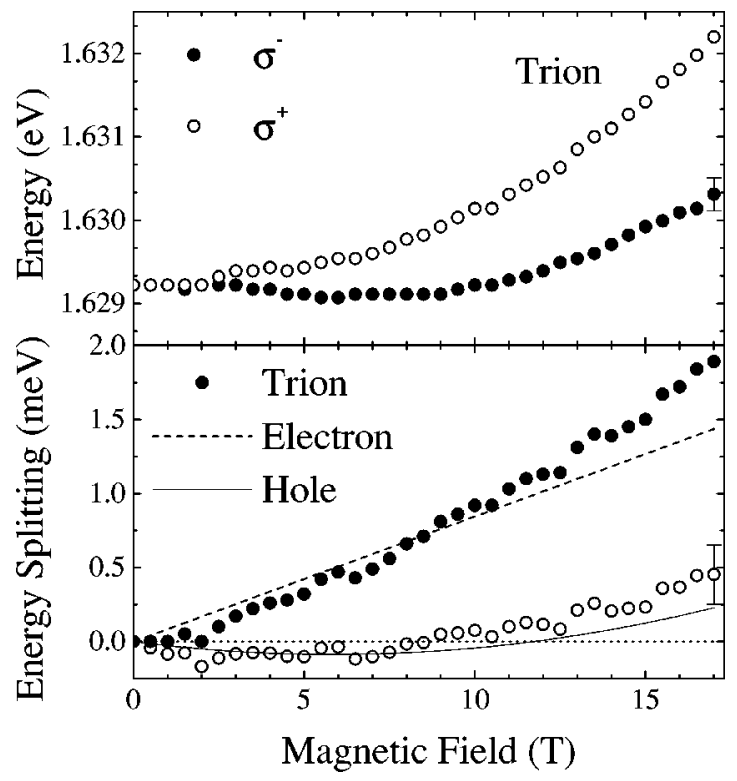

FIG. 4. (a) Energy positions of the trions for $\sigma^{-}$(solid symbols) and $\sigma^{+}$(open symbols) polarizations of sample I. (b) The hole splitting (open symbols) is deduced by subtracting the measured electron splitting (dashed line) from the trion splitting (solid symbols). The solid line curve is the hole splitting as used in the model calculations (see the text).

3(b)]. The two lower exciton levels, which become most populated in a magnetic field (small arrow down), both need an $e \downarrow$ to form a trion. But the magnetic field depopulates this electron level $e \downarrow$ (small arrow up), leading to a reduced probability of trion formation for both $X_{\sigma^{-}}^{-}$and $X_{\sigma^{+}}^{-}$, which results in an increase of the exciton population relative to the trion population.

A comprehensive explanation of the behavior of the trion polarization in the whole range of magnetic fields is more difficult and requires an analysis in terms of a rate equation model including the energy positions as well as the occupancies of the energy levels and the relevant time constants in the system. Before we will perform such an analysis, we will present complementary experimental data obtained by an optically detected resonance technique (ODR). ${ }^{35,36}$

\section{B. Optically detected resonance (ODR)}

The ODR technique detects changes in the PL emission, which are directly related to changes in the occupancies of the exciton, trion, and electron levels that are induced by the absorption of FIR radiation. Figure 5 shows the ODR signal for sample I for different FIR laser wavelengths, listed in order of decreasing laser power: (a) $\lambda_{F I R}=118.8 \mu \mathrm{m}(10.4$ $\mathrm{meV})$, (b) $\lambda_{F I R}=570 \mu \mathrm{m} \quad(2.17 \mathrm{meV})$, and (c) $\lambda_{F I R}$ $=96.5 \mu \mathrm{m}(12.8 \mathrm{meV})$. The shapes of the ODR traces are similar irrespective of the FIR laser line used, and can be divided in three regimes: (1) For $B<5$ T the ODR detected on $X_{\sigma^{-}}^{-}$shows a positive signal, i.e., an increase of the $X_{\sigma^{-}}^{-}$ PL emission, while the $X_{\sigma^{+}}^{-}$shows a negative signal, i.e., a decrease of this PL emission. The $X_{\sigma^{-}}$ODR signal is small and negative in this regime. (2) For $5<B<15 \mathrm{~T}$ the ODR signal shows a broad signal, positive for the trion, in both $\sigma^{-}$ 
and $\sigma^{+}$polarizations, and negative for $X_{\sigma^{-}}$, indicating that the absorption of FIR radiation shifts the equilibrium toward the trion side. In this range, the total PL intensity is constant with varying field, i.e. the sum of the four ODR curves is zero, showing that the FIR radiation induces only changes in the relative occupations of the trion and exciton levels. (3) For higher fields $B>15 \mathrm{~T}$, all ODR curves become zero.

The transition between the first two regimes is most clearly seen in Fig. 5(b) as a kink in the negative slope of the $X_{\sigma^{-}}^{-}$curve at $B \simeq 5 \mathrm{~T}$, accompanied by a change of sign in the $X_{\sigma^{+}}^{-}$ODR curve. It should be noted that the absolute ODR signal of the $X_{\sigma^{+}}$traces is very small, due to the small $X_{\sigma^{+}}$PL emission intensity. However, when these traces are

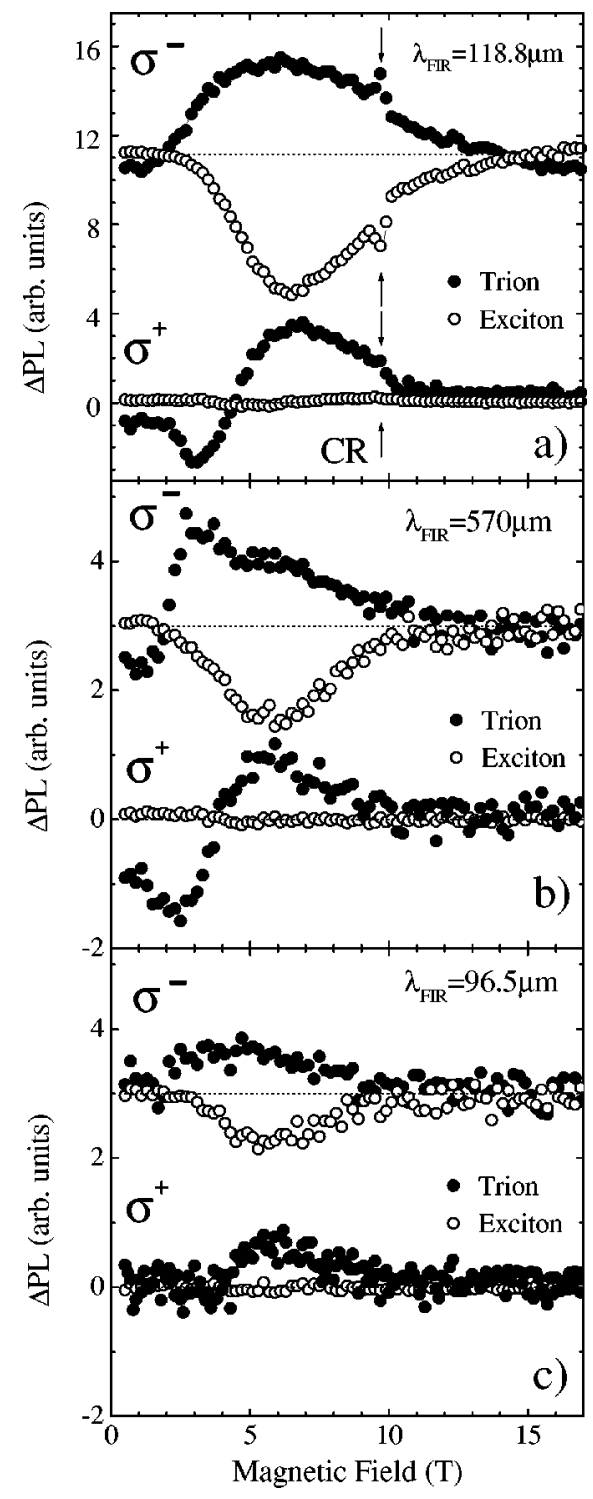

FIG. 5. ODR spectra for three FIR laser lines: (a) $\lambda_{F I R}$ $=118.8 \mu \mathrm{m}$, (b) $\lambda_{F I R}=570 \mu \mathrm{m}$, and (c) $\lambda_{F I R}=96.5 \mu \mathrm{m}$ of sample I with $n_{e}=2 \times 10^{10} \mathrm{~cm}^{-2}$ at $T=1.4 \mathrm{~K}$. A positive signal indicates an increase of PL emission due to the absorption of FIR radiation, a negative signal a decrease. On the spectrum of $\lambda_{F I R}$ $=118.8 \mu \mathrm{m}$ (a) a cyclotron resonance $(\mathrm{CR})$ is visible, indicated by the arrows.

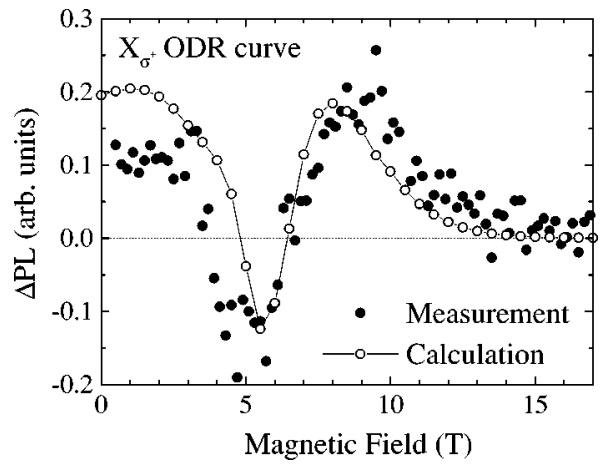

FIG. 6. Magnification of the $X_{\sigma^{+}}$ODR curve for $\lambda_{F I R}$ $=118.8 \mu \mathrm{m}$ (solid symbols) showing a double change of sign: from positive to negative to positive. The open symbols connected by a line are calculations of the ODR signal, which are discussed in Sec. IV B. Note that the scale is the same as used in Fig. 5(a).

enlarged, a double change of sign (from positive to negative to positive) becomes apparent, as is shown for the $118.8-\mu \mathrm{m}$ laser line by the solid symbols in Fig. 6 .

The ODR traces of the 118.8- $\mu m$ laser line [(Fig. 5(a)] show a sharp electronic cyclotron resonance (CR, indicated by arrows) at $9.7 \mathrm{~T}$ (Ref. 44) superimposed on the broad signal. From this resonance field and the wavelength of the FIR laser we obtain an effective electron mass of $m_{e}$ $=0.11 m_{0}$. Both the CR and the broad $\Delta \mathrm{PL}$ signal show an increase of the trion emission (positive signal) and a decrease in exciton emission (negative signal). It is striking, that at $\mathrm{CR}$, where absorption of FIR radiation is expected to be very efficient, the signal is not significantly larger than the $\Delta \mathrm{PL}$ signal. In fact, the largest $\Delta \mathrm{PL}$ signal is found for the $X_{\sigma^{-}}$, where it is $7 \%$ of the peak intensity of the $X_{\sigma^{-}}$PL. Furthermore, apart from the CR we do not see other resonant features such as intra-exciton or intra-trion transitions, which are reported for GaAs based structures. ${ }^{33,36,44,45}$

The broad $\Delta \mathrm{PL}$ signal is attributed to non-resonant heating, because of the following reasons: (i) The signal is similar for all FIR laser lines used (Fig. 5) and has the same sign as the CR, which is unambiguously due to heating of the electron system. The differences in intensity are mainly due to different FIR laser power $\left(P_{118.8 \mu \mathrm{m}}>P_{570 \mu \mathrm{m}}>P_{96.5 \mu \mathrm{m}}\right)$. (ii) The signal is found to dependent linearly on the FIR laser power (not shown). (iii) It diminishes when the temperature of the system is increased from $T=1.4 \mathrm{~K}$ to $T=4.2 \mathrm{~K}$ (not shown). (iv) The shape of the signal is similar to that observed on a similar sample, but using microwave radiation with energy of about $0.3 \mathrm{meV}$ instead of FIR radiation. ${ }^{33,34}$

\section{DISCUSSION}

The precise magnetic field dependence of the PL intensity and polarization of the exciton and trion levels and the FIR induced changes herein (ODR signal) is a complicated function of many parameters, such as the field-dependent energy level diagram and the characteristic time constants of the exciton-trion-electron system. In order to explain the MPL and ODR data we have used a more or less standard rate 


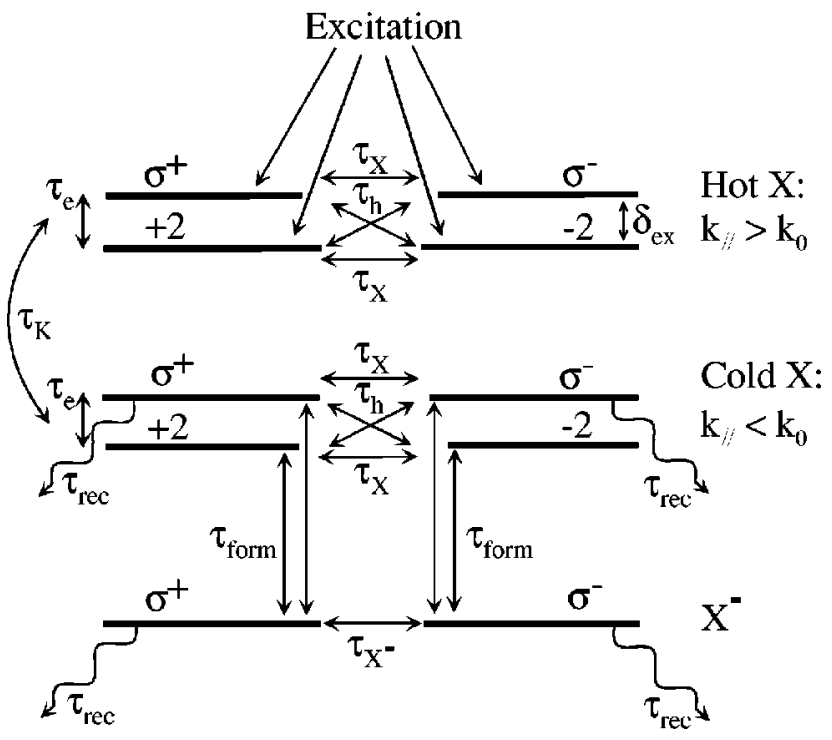

FIG. 7. Schematic representation of the rate equation model in a magnetic field (see text for a detailed description). For reasons of clarity the energy levels are shown for the case $B=0 \mathrm{~T}$. In a magnetic field the levels become split, as shown in Fig. 8 .

equation model ${ }^{23,30}$ adapted to the magnetic field case, which incorporates the experimentally obtained values for the energy levels and all relevant time constants associated to several processes, such as trion formation, spin-flip scattering, and radiative recombination. In this section we will first present the rate equation model, followed by the modeling results of both the MPL and the ODR data.

\section{A. Rate equation model}

The rate equation model, schematically shown in Fig. 7, includes four hot exciton levels $\left(k_{/ /}>k_{0}\right)$, four cold excitons levels $\left(k_{/ /}<k_{0}\right)$, two of which can recombine under emission of circularly polarized light, and two singlet trion levels which also emit circularly polarized light. The zero-field exchange splitting, which splits of the dark $+/-2$ excitons ${ }^{46,47}$ toward lower energy with respect to the bright excitons, is taken into account by introducing a parameter $\delta_{e x}$ in the model calculations which was varied in the range of $0-0.2$ meV. The energy level diagram as shown in Fig. 7 holds for the $B=0 \mathrm{~T}$ case. In a magnetic field the exciton and trion levels, and the electron levels that are not shown in the figure, are split (see Fig. 8) due to the Zeeman effect.

The processes that are included in the model are denoted by the arrows. A double arrow indicates that also the reversed process is taken into account. To mimic the experimental situation in which excitons are excited nonresonantly, we introduce excitons in high $k_{/ /}$states (hot excitons ${ }^{5}$ ), which cannot recombine since they are outside the lightcone, but can flip their spin (electron $\tau_{e}$, hole $\tau_{h}$, or both $\tau_{X}$ ) before relaxation $\left(\tau_{K}\right)$ to the bottom of the band to cold or low $k_{/ /}$-value excitons. The cold excitons can flip their spin $\left(\tau_{e}, \tau_{h}\right.$, or $\left.\tau_{X}\right)$, can be excited to the $k_{/ /}>k_{0}$ region $\left(\tau_{K}^{*}\right)$, can recombine $\left(\tau_{\text {rec }}\right)$ or can form a trion $\left(\tau_{\text {form }}\right)$ for which they need an electron with the proper spin.

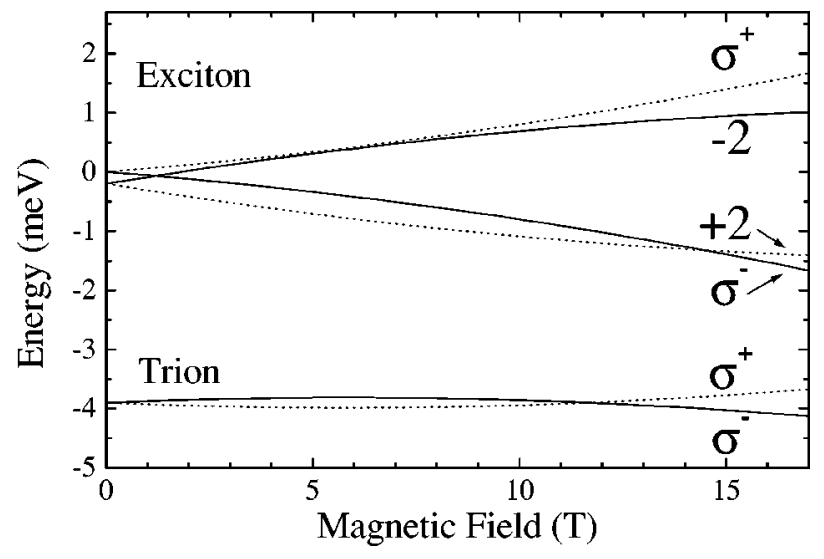

FIG. 8. The magnetic field dependence of the exciton and trion levels, determined from the PL measurements (also see Fig. 4), as they are used in the model calculations. An exchange splitting of $0.2 \mathrm{meV}$ is included.

The trions can also flip their spin $\left(\tau_{X^{-}}\right)$, dissociate into an exciton $\left(\tau_{\text {form }}^{*}\right)$ or recombine $\left(\tau_{\text {rec }}\right)$. The model includes the formation processes indicated in Fig. 3, thereby assuming that the spin orientation of the electron and hole in the exciton is conserved. ${ }^{23}$ The electrons are taken to be thermally distributed over the quantized Landau levels, which are Gaussian broadened with a width of $0.25 \mathrm{meV}$. Furthermore, in the model we assume that only excitons within the lightcone can $\left(k_{/ /}<k_{0}\right)$ can form a trion, although results from a previous paper ${ }^{41}$ showed that this restriction might be too severe. However, allowing the hot excitons $\left(k_{/ /}>k_{0}\right)$ to form trions effects only the ratio of the total exciton emission to the total trion emission $\left(I_{X} / I_{X^{-}}\right)$, and not the relative distributions among the exciton and among the trion levels, which are of importance in the MPL and ODR data considered here.

To describe the system, ten coupled rate equations are used similar to the one shown here for $n_{X}^{\sigma^{-}}$,

$$
\begin{aligned}
\frac{\mathrm{d} n_{X}^{\sigma^{-}}}{\mathrm{d} t}= & n_{X_{h o t} \sigma_{K}^{-}} \tau_{K}^{-1}-n_{X}^{\sigma^{-}} \tau_{K}^{*-1}-\frac{n_{X}^{\sigma^{-}} n_{e}^{\downarrow}}{n_{X}^{\sigma^{-}}+n_{e}^{\downarrow}} \tau_{\text {form }}^{-1}+n_{X^{-}}^{\sigma^{-}} \tau_{\text {form }}^{*-1} \\
& -n_{X}^{\sigma^{-}}\left(\tau_{\text {rec }}^{-1}+\tau_{X}^{*-1}+\tau_{e}^{*-1}+\tau_{h}^{-1}\right)+n_{X}^{\sigma^{+}} \tau_{X}^{-1} \\
& +n_{X}^{-2} \tau_{e}^{-1}+n_{X}^{+2} \tau_{h}^{*-1}
\end{aligned}
$$

where the densities are denoted as $n_{j}^{i}$, where $j=X, X_{\text {hot }}$, $X^{-}, e$ and $i=\sigma^{+/-},+/-2, \downarrow / \uparrow$. The terms containing the time parameters $\tau_{k}^{-1}\left(k=r e c\right.$, form $, K, X, X^{-}, e$, or $\left.h\right)$ denote the forward processes, i.e., toward lower energy, and the terms containing $\tau_{k}^{*-1}$ denote the reversed processes, i.e., toward higher energy.

The large number of parameters that can be varied is significantly reduced by several conditions that we impose upon the model. In the limiting case that the recombination times are infinite, the system should establish a chemical equilibrium between the exciton and trion levels, and a thermal equilibrium among the hot exciton levels, among the cold exciton levels and among the trion levels. The tendency of 
the system to reach a chemical equilibrium is represented by relating the trion formation time $\left(\tau_{\text {form }}\right)$ and its reverse $\left(\tau_{\text {form }}^{*}\right)$ as

$$
\frac{\tau_{\text {form }}}{\tau_{\text {form }}^{*}}=\frac{k_{B} T}{E_{F}} \frac{m_{X}}{m_{X^{-}}} \exp \left[-E_{b} / k_{B} T\right],
$$

where $m_{X}$ and $m_{X^{-}}$are the exciton and trion masses, and $E_{F}$ is the Fermi energy of the 2DEG in the quantum well. The attempt of the system to reach thermal equilibrium is represented by relating the time parameters $\tau_{k}$ and $\tau_{k}^{*}\left(k=X, X^{-}\right.$, $e$, or $h)$ by a Boltzman factor ${ }^{23,30}: \tau_{k}^{-1} / \tau_{k}^{*-1}=\exp$ $\left(-\Delta E / k_{B} T\right)$, where $\Delta E$ is the energy splitting of the two corresponding levels. To relate the relaxation times $\tau_{K}$ and $\tau_{K}^{*}$, we take the energy of the lightcone $E_{\text {lightcone }}=0.07 \mathrm{meV}$ (Ref. 23) for the energy splitting $\Delta E$. Furthermore, for the sake of clarity we assume $\tau_{\text {rec }}^{X} \sim \tau_{\text {rec }}^{X^{-}}=\tau_{\text {rec }}$, and we have verified that the calculations are relatively insensitive to changes $(\sim$ factor of 2$)$ in the ratio $\tau_{\text {rec }}^{X} / \tau_{\text {rec }}^{X^{-}}$. Finally, the time constants of the system are defined relative to the trion formation time $\tau_{\text {form }}$, and are taken to be constant with the temperature and the magnetic field.

In the rate equations we use the phenomenological term $n_{X}^{i} n_{e}^{\downarrow / \uparrow} /\left(n_{X}^{i}+n_{e}^{\downarrow / \uparrow}\right) \tau_{\text {form }}^{-1}$ to determine the trion formation rate, where $i=\sigma^{+/-},+/-2$ and $\downarrow / \uparrow$ is a proper combination which forms a singlet trion (see Fig. 3). This term ensures that the rate properly depends on the electron density similar to a true chemical equilibrium. In the case of a large electron density $\left(n_{e}^{\downarrow / \uparrow} \gg n_{X}^{i}\right)$, the trion formation rate equals $n_{X}^{i} \tau_{\text {form }}^{-1}$, and is primarily sensitive to the changes in the exciton density. In the opposite case, i.e., a small electron density $\left(n_{e}^{\downarrow / \uparrow} \ll n_{X}^{i}\right)$, the rate is equal to $n_{e}^{\downarrow / \uparrow} \tau_{\text {form }}^{-1}$, and depends mainly on the electron density. Moreover, in case of zero $n_{e}^{\downarrow / \uparrow}$, i.e., when there are no electrons left for trion formation, the trion formation rate reduces to zero, as required.

We calculate the state of the dynamical equilibrium for the system numerically, by letting the system develop iteratively in small steps $\Delta t$ until it reaches a steady-state solution $(d n / d t=0)$ for all ten equations. Before starting to describe the experimental data, we have checked that the mentioned boundary conditions for the model are fulfilled. The calculations are done at a fixed temperature, assuming that the trions, excitons, and electrons are at (or close to) the bath temperature. In the samples containing a background electron density, the 2DEG acts as a cold buffer that efficiently cools the excitons. Possible cooling mechanisms are as follows: (1) the excitons cool down as a whole through scattering processes with the 2DEG, and (2) cold excitons are created from a cold 2DEG electron and a photoexcited hole that is efficiently cooled due to spin-orbit interaction. Moreover, we find that the polarization behavior of the trion and exciton emission in a magnetic field becomes more pronounced when lowering the temperature to $T=1.4 \mathrm{~K}$. The magnitude of the ODR signal shows an increase when lowering the temperature from $T=4.2$ to $1.4 \mathrm{~K}$. Therefore, the assumption that the excitons and trions are at the lattice temperature seems to be reasonable.

\section{B. Results of the model calculations}

The final aim of the model calculations was to find a unique set of parameters that simultaneously describes the polarized MPL and ODR data in order to unravel the importance of the different processes for the dynamical equilibrium in the exciton-trion-electron system. It is important to note that in this respect the ODR data plays a crucial role. It turned out that the polarized MPL data can be described reasonably well using two other models, which are more simple than the full model described above, but which can be disregarded because they are not able to reproduce the ODR data. These two models make use of either a thermal equilibrium between the exciton and trion states (not shown here) or a rate equation model that excludes the presence of hot and dark excitons. ${ }^{48}$ In this section we focus on the results of the full model calculations. The input parameters of the model calculation can be divided into two distinct classes, that is (1) the positions of the energy levels, and (2) the time constants. The energy positions of the exciton and trion levels were determined from the experiments, namely from the field dependent PL peak positions (Fig. 4). This analysis resulted in the energy position scheme for the excitons and trions depicted in Fig. 8. Note that up to $12 \mathrm{~T}$ the lowest trion level corresponds to a $\sigma^{+}$polarization, whereas for higher fields the $\sigma^{-}$trion level is the lowest in energy; as a consequence of the hole splitting (Fig. 4). In Fig. 8 we show the energy scheme for the case $\delta_{\text {exc }}=0.2 \mathrm{meV}$, which makes the $X_{+2}$ level the lowest in energy up to $15 \mathrm{~T}$, after which the $X_{\sigma^{-}}$becomes the lowest energy level. The actual magnetic field where the lowest exciton level changes its nature depends obviously on the value of the exchange splitting which was varied in the range of $0-0.2 \mathrm{meV}$ in order to get the best fit of the MPL and ODR data.

As a starting set of time constants we have adopted the values found in time-resolved studies, ${ }^{23,30}$ and allowed changes up to a factor of 10 larger or smaller during the optimization process. We have assumed that the emission intensity of a specific transition is proportional to the occupation of that level divided by the corresponding recombination time. Using this procedure the polarization degree of the different (charged) exciton levels was calculated for a given set of time constants (Fig. 9) at a fixed temperature ( $T$ $=1.4 \mathrm{~K})$ and electron density $\left(n_{e}=2 \times 10^{10} \mathrm{~cm}^{-2}\right)$, which corresponds to the measurements performed on sample I. Using the same set, the ODR traces are simulated by subtracting the PL intensities calculated for $T=1.4 \mathrm{~K}$ from those calculated for $T=1.45 \mathrm{~K}$ (Fig. 10). Figure 9(a) (solid symbols) and Fig. 10(a) display the optimized results of the model calculations for the polarized PL data and ODR traces respectively. The most important features of both PL polarization [compare Fig. 9(a) with Fig. 2(b)] and ODR [compare Fig. 10(a) with Fig. 5, and compare the two curves in Fig. 6] are reproduced using the following time constants: $\tau_{\text {form }} \sim 0.2 \tau_{\text {rec }} \sim \tau_{K} \sim 10 \tau_{X} \sim \tau_{X^{-}} \sim \tau_{e} \sim \tau_{h}$. These values are indeed consistent with the time constants obtained from time-resolved ${ }^{23,30}$ and cw (Ref. 49) experiments. Note that although for simplicity reasons these time constants are taken to be independent of the magnetic field, the transition 
a)

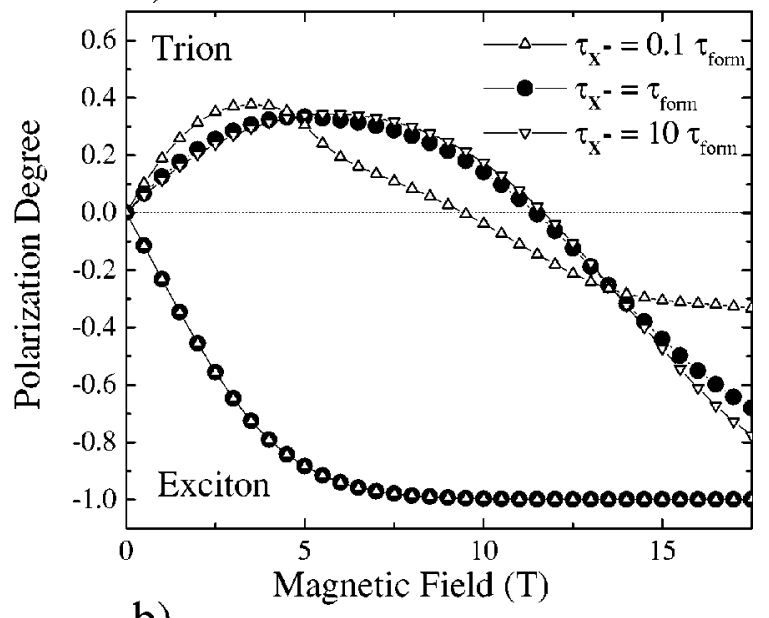

b)

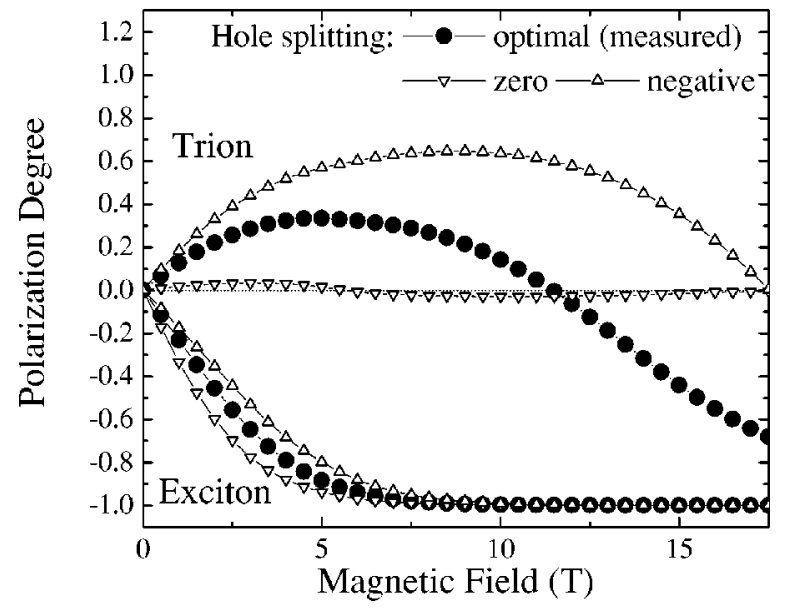

FIG. 9. Model calculations of the trion and exciton polarization degree (solid symbols). (a) Dependence of the calculations on the trion flip time. (b) Dependence of the calculations on the hole spin splitting (see text for details).

rates between the levels vary with the field, showing that the processes are driven by the field dependent occupancies of the energy levels. The calculations were found to be weakly dependent on the exchange splitting. The optimized value was determined to be in the range $\delta_{e x}=0.1-0.2 \mathrm{meV}$, which is consistent with the findings of Vanelle et $_{\text {al }}{ }^{23}$ for CdTebased QW's.

We have also verified the sensitivity of the outcome of the model with respect to variations in the other input parameters. We have found that the trion polarization degree and in particular the cross-over field at which it turns negative is not very sensitive to the values used for the time constants. Fig. 9a shows for instance the influence of making the trion spin-flip time $\tau_{X^{-}}$ten times higher or lower than $\tau_{\text {form }}$. Varying the other time constants leads to similar results. Conversely, $\mathcal{P}$ depends strongly on the trion energy splitting which is determined by the hole spin splitting. Figure 9(b) shows calculations of the polarization degree for zero hole splitting (down triangles), for the optimal hole splitting (solid circles), and for a hole splitting that is negative up to $B \sim 17.5 \mathrm{~T}$ before turning positive (up triangles). It
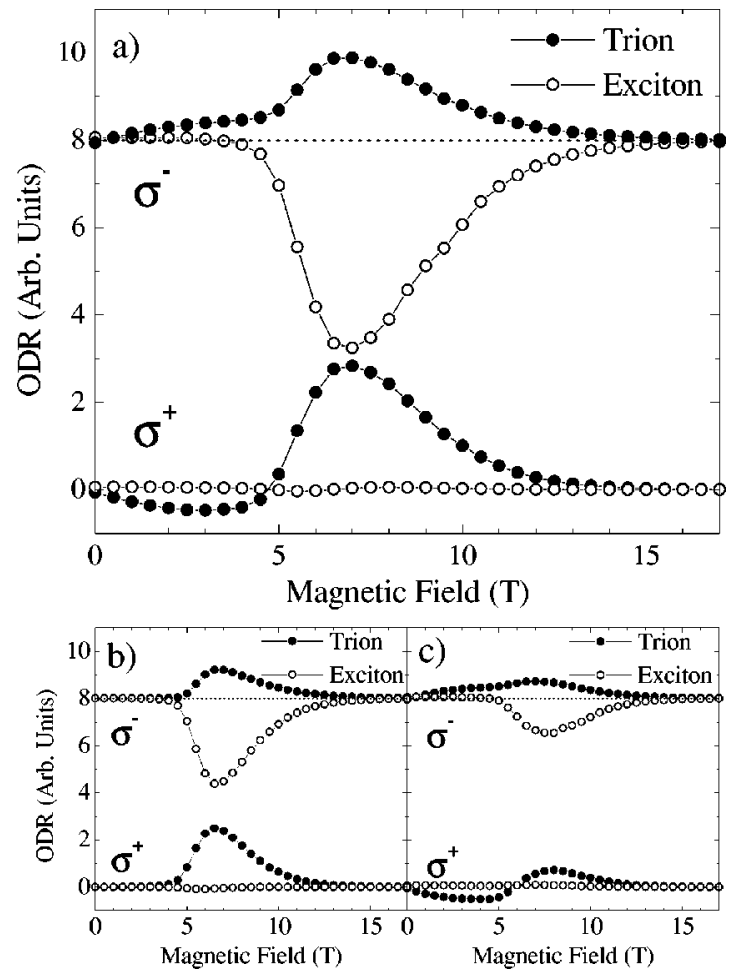

FIG. 10. (a) Model calculations describing the ODR spectrum by subtracting calculations done at $T=1.4 \mathrm{~K}$ from calculations done at $T=1.45 \mathrm{~K}$. Calculations performed by raising the temperature of (b) only the electron system and (c) only the exciton-trion system.

is clearly seen that the polarization degree is best described by the solid symbols, using the hole splitting which is shown in Fig. 4 as a solid line and is indeed very close to the experimentally determined behavior.

From these modeling results we can identify two mechanisms that lead to the positive trion polarization degree at low magnetic field as is observed experimentally: (1) Up to $B=12 \mathrm{~T}, X_{\sigma^{+}}^{-}$is the lowest trion level (see Fig. 8), favoring the relaxation to this state which results in a positive $\mathcal{P}$. This is the dominant mechanism which leads to a change of sign in $\mathcal{P}$ at $B=12 \mathrm{~T}$ when $X_{\sigma^{-}}^{-}$becomes lowest in energy. (2) For $B \leqslant 14 \mathrm{~T}$, the occupation of the $X_{+2}$ level will be higher than that of the $X_{\sigma^{-}}$, not only because it is lower in energy (Fig. 8), but also because it is a nonradiative state in contrast to the radiative $X_{\sigma^{-}}$state. The formation rate of $X_{\sigma^{+}}^{-}$out of $X_{+2}$ and $e \downarrow$ [left dotted arrow in Fig. 3(a)] will therefore be much larger than that of $X_{\sigma^{-}}^{-}$out of $X_{\sigma^{-}}$and $e \downarrow$ (right solid arrow), resulting in a larger density of $X_{\sigma^{+}}^{-}$than $X_{\sigma^{-}}^{-}$, i.e. a positive polarization degree. The contribution of $X_{\sigma^{+}}$and $X_{-2}$ to the trion formation is negligible because these levels depopulate fastly as shown by the negative polarization degree of the excitons. For higher fields, $B>14 \mathrm{~T}$, besides the fact that $X_{\sigma^{-}}^{-}$becomes lower in energy, also $X_{\sigma^{-}}$becomes lower in energy (Fig. 8) resulting in an enhanced formation of $X_{\sigma^{-}}^{-}$(right solid arrow) leading to the observed negative polarization degree. 
Figure 10(a) reveals that the calculations describe the general trends of the ODR traces correctly for low $(B<5 \mathrm{~T})$, moderate $(5<B<15 \mathrm{~T})$ and high $(B>15 \mathrm{~T})$ fields, and allow us to reveal the different mechanisms leading to the signals in these regimes. For $B<5 \mathrm{~T}$, the $X_{\sigma^{+}}^{-}$curve shows a negative signal and the $X_{\sigma^{-}}^{-}$curve a positive signal, while the exciton curves show a negligible signal. For $5<B<15 \mathrm{~T}$, we calculate a positive signal for both $X_{\sigma^{+}}^{-}$and $X_{\sigma^{-}}^{-}$and a negative signal for the $X_{\sigma^{-}}$curve, and for $B>15 \mathrm{~T}$ the calculated curves are zero. The $X_{\sigma^{+}}$curve is very small, but there is a double change of sign (from positive to negative to positive) as could also be observed in the measurements (see open symbols in Fig. 6).

The fact that a small temperature increase of $0.05 \mathrm{~K}$ is sufficient to describe the ODR data confirms that the ODR signal can be attributed to nonresonant heating, as discussed above. Moreover, the calculations can make a distinction between the effect caused by raising only the electron temperature while the exciton and trion temperature remains at $T$ $=1.4 \mathrm{~K}$ [Fig. 10(b)] and raising only the exciton and trion temperature while the electron temperature remains at $T$ $=1.4 \mathrm{~K}$ [Fig. 10(c)]. It is clear that the low magnetic field signal is completely determined by heating of the excitons and trions, while the high magnetic field effect is dominated by the electron heating. Whereas FIR-induced heating of the 2DEG is a more or less regular phenomenon, heating of the excitons and trions by FIR radiation is less common. ${ }^{33}$ The excitons and trions can be heated by scattering with 2DEG electrons at an elevated temperature or during the trion formation process using a FIR heated electron. Alternatively, the exciton and trion heating can originate from the dark excitons, which can absorb FIR radiation because of their very long lifetime, in contrast to the radiative excitons and trions which have much smaller probability to absorb FIR radiation since their lifetime is too short ( $\sim 100 \mathrm{ps})$. We conclude that the presence of the ODR signal at low magnetic fields is a direct consequence of the presence of dark excitons in the system, and their significant contribution to the trion formation process. Calculations excluding dark excitons in the system indeed show no low field ODR signal. ${ }^{48}$

Based on the nonresonant heating mechanism, the response of the PL emission on the FIR can also be understood qualitatively by considering the occupancy of the energy levels in the magnetic field (Fig. 3). For the $B<5 \mathrm{~T}$ regime the $X_{+2}$ level is more occupied than the $X_{\sigma^{-}}$level leading to more $X_{\sigma^{+}}^{-}$formation relative to $X_{\sigma^{-}}^{-}$formation, as discussed above. The FIR induced heating promotes $X_{+2}$ to the $X_{\sigma^{-}}$ level leading to an increased formation of $X_{\sigma^{-}}^{-}$(positive signal) at the expense of $X_{\sigma^{+}}^{-}$(negative signal). In the regime $5<B<15 \mathrm{~T}$ the ODR signal is mainly a result of heating of the electron system. As illustrated in Fig. 3(b), the magnetic field polarizes the electron and exciton population reducing the trion formation. Heating of the system by the absorption of FIR radiation reduces the electron polarization, i.e., partially canceling the small arrow up in Fig. 3(b), leading to an increase of trion formation (positive signal for both $X_{\sigma^{-}}^{-}$and $X_{\sigma^{+}}^{-}$) at the expense of the exciton population (negative sig- nal of $X_{\sigma^{-}}$). For higher fields $B>15 \mathrm{~T}$, heating of the system by FIR radiation no longer has any effect on the PL intensities of excitons and trions, resulting in no ODR signal for all curves.

When analyzing the calculated rates in detail, we find that the $X_{\sigma^{+}}$ODR curve (Fig. 6) can be explained qualitatively by considering three effects that interact in a subtle manner. First, the $X_{\sigma^{+}}$occupation is enhanced by a heating induced redistribution of the excitons among the exciton levels. This effect is in competition with a second effect, namely, that the trion formation channel $X_{\sigma^{+}}+e \uparrow \rightarrow X_{\sigma^{+}}^{-}$becomes more efficient, which reduces the $X_{\sigma^{+}}$population. For the range $B$ $<4 \mathrm{~T}$, the first effect is dominant resulting in a positive ODR signal. For $4<B<6.5 \mathrm{~T}$, the latter effect is stronger leading to a negative ODR signal. For higher fields, $B$ $>6.5 \mathrm{~T}$, the FIR radiation promotes electrons from the $e \uparrow$ level to the $e \downarrow$ level, as discussed before, leading to a less efficient $X_{\sigma^{+}}+e \uparrow \rightarrow X_{\sigma^{+}}^{-}$formation channel. This third effect counteracts the second effect, and therefore we observe a positive ODR signal in this range. Note that these effects are very small compared to the effects considered in explaining the other ODR curves.

\section{CONCLUSION}

We have presented $\mathrm{cw}$ PL and ODR measurements as a function of the magnetic field, which show that the formation of trions out of excitons and electrons can be regarded as a dynamical equilibrium, consisting of a chemical equilibrium modified by finite recombination times and spin-flip times. This result elucidates the discrepancy between cw experiments, where the formation is often described as a chemical equilibrium between trions, excitons, and electrons, and time-resolved experiments, where formation times, recombination times, and spin-flip times are found to be of the same order of magnitude. The ODR measurements reveal the presence of the dark excitons in the system, and show that they contribute significantly to the trion formation. The magneticfield-induced polarization degree of the trion is found to be mainly determined by the heavy-hole spin splitting in the sample.

We have incorporated the formation process in a rate equation model, which includes spin-split, field-dependent energy levels, as well as formation times, recombination times and spin-flip times between the trion (exciton) levels. This model successfully describes the presented PL and ODR experimental data using one unique set of parameters, $\tau_{\text {form }} \sim 0.2 \tau_{\text {rec }} \sim \tau_{K} \sim 10 \tau_{X} \sim \tau_{X}-\sim \tau_{e} \sim \tau_{h}$, which is consistent with results from time-resolved experiments. To explain our data, it is sufficient to take the characteristic times of the system to be independent of the magnetic field. The varying rates with field are found to be merely determined by the field-dependent occupancies of the spin-split energy levels.

\section{ACKNOWLEDGMENTS}

This work was supported by the "Stichting voor Fundamenteel Onderzoek" (FOM) financially supported by NWO 
(the Netherlands), by the Deutsche Forschungsgemeinschaft (grants Os98/6 and SFB 410, Germany), and by the Center of Excellence CELDIS established under EU Contract No. ICA1CT2000-70018 (Poland).
${ }^{1}$ M.A. Lambert, Phys. Rev. Lett. 1, 450 (1958).

${ }^{2}$ K. Kheng, R.T. Cox, Y. Merle d'Aubigné, F. Bassani, K. Saminadayar, and S. Tatarenko, Phys. Rev. Lett. 71, 1752 (1993).

${ }^{3}$ A.J. Shields, M. Pepper, D.A. Ritchie, M.Y. Simmons, and G.A.C. Jones, Phys. Rev. B 51, 18049 (1995).

${ }^{4}$ A.J. Shields, J.L. Osborne, M.Y. Simmons, M. Pepper, and D.A. Ritchie, Phys. Rev. B 52, R5523 (1995).

${ }^{5}$ G. Finkelstein, V. Umansky, I. Bar-Joseph, V. Ciulin, S. Haacke, J.-D. Ganière, and B. Deveaud, Phys. Rev. B 58, 12637 (1998).

${ }^{6}$ G.V. Astakhov, D.R. Yakovlev, V.P. Kochereshko, W. Ossau, J. Nürnberger, W. Faschinger, and G. Landwehr, Phys. Rev. B 60, R8485 (1999).

${ }^{7}$ A.J. Shields, M. Pepper, M.Y. Simmons, and D.A. Ritchie, Phys. Rev. B 52, 7841 (1995).

${ }^{8}$ D.M. Whittaker and A.J. Shields, Phys. Rev. B 56, 15185 (1997).

${ }^{9}$ G. Yusa, H. Shtrikman, and I. Bar-Joseph, Phys. Rev. Lett. 87, 216402 (2001).

${ }^{10}$ C. Riva, F.M. Peeters, and K. Varga, Phys. Rev. B 63, 115302 (2001).

${ }^{11}$ T. Vanhoucke, M. Hayne, M. Henini, and V.V. Moshchalkov, Phys. Rev. B 63, 125331 (2001).

${ }^{12}$ D.R. Yakovlev, G.V. Astakhov, W. Ossau, S.A. Crooker, K. Uchida, N. Miura, A. Waag, N.A. Gippius, A.Yu. Sivachenko, and A.B. Dzyubenko, Phys. Status Solidi B 227, 353 (2001).

${ }^{13}$ G.V. Astakhov, D.R. Yakovlev, V.P. Kochereshko, W. Ossau, W. Faschinger, J. Puls, F. Henneberger, S.A. Crooker, Q. McCulloch, D. Wolverson, N.A. Gippius, and A. Waag, Phys. Rev. B 65, 165335 (2002).

${ }^{14}$ B. Stébé, G. Munschy, L. Stauffer, F. Dujardin, and J. Murat, Phys. Rev. B 56, 12454 (1997).

${ }^{15}$ T. Wojtowicz, M. Kutrowski, G. Karczewski, and J. Kossut, Acta Phys. Pol. A 94, 199 (1998).

${ }^{16}$ V. Huard, R.T. Cox, K. Saminadayar, A. Arnoult, and S. Tatarenko, Phys. Rev. Lett. 84, 187 (2000).

${ }^{17}$ D.R. Yakovlev, V.P. Kochereshko, R.A. Suris, H. Schenk, W. Ossau, A. Waag, G. Landwehr, P.C.M. Christianen, and J.C. Maan, Phys. Rev. Lett. 79, 3974 (1997).

${ }^{18}$ G. Finkelstein, H. Shtrikman, and I. Bar-Joseph, Phys. Rev. Lett. 74, 976 (1995).

${ }^{19}$ G. Eytan, Y. Yayon, M. Rappaport, H. Shtrikman, and I. BarJoseph, Phys. Rev. Lett. 81, 1666 (1998).

${ }^{20}$ F. Pulizzi, W.H.A. Thijssen, P.C.M. Christianen, J.C. Maan, D.R. Yakovlev, W. Ossau, T. Wojtowicz, G. Karczewski, and J. Kossut, Proceedings of Semimag 14, Matsue [Physica B 298, 397 (2001)].

${ }^{21}$ D. Sanvitto, F. Pulizzi, A.J. Shields, P.C.M. Christianen, S.N. Holmes, M.Y. Simmons, D.A. Ritchie, J.C. Maan, and M. Pepper, Science 294, 837 (2001).

${ }^{22}$ D.R. Yakovlev, J. Puls, G.V. Mikhailov, G.V. Astakhov, V.P. Kochereshko, W. Ossau, J. Nürnberger, W. Faschinger, F. Henneberger, and G. Landwehr, Phys. Status Solidi A 178, 501 (2000).
${ }^{23}$ E. Vanelle, M. Paillard, X. Marie, T. Amand, P. Gilliot, D. Brinkmann, R. Lévy, J. Cibert, and S. Tatarenko, Phys. Rev. B 62, 2696 (2000).

${ }^{24}$ D. Sanvitto, R.A. Hogg, A.J. Shields, D.M. Whittaker, M.Y. Simmons, D.A. Ritchie, and M. Pepper, Phys. Rev. B 62, R13 294 (2000).

${ }^{25}$ V. Ciulin, P. Kossacki, S. Haacke, J.-D. Ganière, B. Deveaud, A. Esser, M. Kutrowski, and T. Wojtowicz, Phys. Rev. B 62, R16 310 (2000).

${ }^{26}$ A. Ron, H.W. Yoon, M.D. Sturge, A. Manassen, E. Cohen, and L.N. Pfeiffer, Solid State Commun. 97, 741 (1996).

${ }^{27}$ A. Manassen, E. Cohen, A. Ron, E. Linder, and L.N. Pfeiffer, Phys. Rev. B 54, 10609 (1996).

${ }^{28}$ J. Siviniant, D. Scalbert, A.V. Kavokin, D. Coquillat, and J.-P. Lascaray, Phys. Rev. B 59, 1602 (1999).

${ }^{29}$ A. Esser, E. Runge, R. Zimmermann, and W. Langbein, Phys. Status Solidi A 178, 489 (2000).

${ }^{30}$ A. Vinattieri, J. Shah, T.C. Damen, D.S. Kim, L.N. Pfeiffer, M.Z. Maialle, and L.J. Sham, Phys. Rev. B 50, 10868 (1994).

${ }^{31}$ D. R. Yakovlev, V. P. Kochereshko, W. Ossau, G. Landwehr, P. C. M. Christianen, J. C. Maan, T. Wojtowicz, G. Karczewski, and J. Kossut, Proceedings ICPS-24, Jerusalem (1998).

${ }^{32}$ D.R. Yakovlev, H.A. Nickel, B.D. McCombe, A. Keller, G.V. Astakhov, V.P. Kochereshko, W. Ossau, J. Nürnberger, W. Faschinger, and G. Landwehr, J. Cryst. Growth 214/215, 823 (2000).

${ }^{33}$ C.Y. Hu, W. Ossau, D.R. Yakovlev, G. Landwehr, T. Wojtowicz, G. Karczewski, and J. Kossut, Phys. Rev. B 58, R1766 (1998).

${ }^{34}$ W. Ossau, D.R. Yakovlev, C.Y. Hu, V.P. Kochereshko, G.V. Astakhov, R.A. Suris, P.C.M. Christianen, and J.C. Maan, Fiz. Tverd. Tela (St. Petersburg) 41, 831 (1999) [Phys. Solid State 41, 751 (1999)].

${ }^{35}$ M.S. Salib, H.A. Nickel, G.S. Herold, A. Petrou, B.D. McCombe, R. Chen, K.K. Bajaj, and W. Schaff, Phys. Rev. Lett. 77, 1135 (1996).

${ }^{36}$ H.A. Nickel, G.S. Herold, T. Yeo, G. Kioseoglou, Z.X. Jiang, B.D. McCombe, A. Petrou, D. Broido, and W. Schaff, Phys. Status Solidi B 210, 341 (1998).

${ }^{37}$ T. Wojtowicz, M. Kutrowski, G. Karczewski, and J. Kossut, Appl. Phys. Lett. 73, 1379 (1998).

${ }^{38}$ G.V. Astakhov, V.P. Kochereshko, D.R. Yakovlev, W. Ossau, J. Nürnberger, W. Faschinger, G. Landwehr, T. Wojtowicz, G. Karczewski, and J. Kossut, Phys. Rev. B 65, 115310 (2002).

${ }^{39}$ In reflection the polarization of the trion is equal to the electron polarization (Refs. 2,3, and 6), which at a given field is determined by the electron $g$ factor [measured (Ref. 43)] and the electron density, which thus can be determined.

${ }^{40} \mathrm{~F}$. Reiff, Fundamentals of statistical and thermal physics (McGraw-Hill, Singapore, 1984) p. 322.

${ }^{41}$ C. R. L. P. N. Jeukens, P. C. M. Christianen, J. C. Maan, D. R. Yakovlev, W. Ossau, T. Wojtowicz, G. Karczewski, and J. Kos- 
sut, Proceedings of OECS7, Montpellier [Phys. Status Solidi A 190, 813 (2002)].

${ }^{42}$ K. Kheng, R.T. Cox, V.P. Kochereshko, K. Saminadayar, S. Tatarenko, F. Bassani, and A. Franciosi, Superlatt. Microstruct. 15, 253 (1994).

${ }^{43}$ A.A. Sirenko, T. Ruf, M. Cardona, D.R. Yakovlev, W. Ossau, A. Waag, and G. Landwehr, Phys. Rev. B 56, 2114 (1997).

${ }^{44}$ C. J. Meining, M. Furis, H. A. Nickel, D. R. Yakovlev, W. Ossau, A. Petrou, and B. D. McCombe, Proceedings ICPS-25, Osaka (2000).

${ }^{45}$ A.B. Dzyubenko, A.Yu. Sivachenko, H.A. Nickel, T.M. Yeo, G.
Kioseoglou, B.D. McCombe, and A. Petrou, Physica E 6, 156 (2000).

${ }^{46}$ H.W. van Kesteren, E.C. Cosman, W.A.J.A. van der Poel, and C.T. Foxon, Phys. Rev. B 41, 5283 (1990).

${ }^{47}$ Yu.G. Kusrayev, B.P. Zakharchenya, G. Karczewski, T. Wojtowicz, and J. Kossut, Solid State Commun. 104, 465 (1997).

${ }^{48}$ C. R. L. P. N. Jeukens, P. C. M. Christianen, J. C. Maan, D. R. Yakovlev, W. Ossau, T. Wojtowicz, G. Karczewski, and J. Kossut, Proceedings ICPS-25, Osaka (2000).

${ }^{49}$ M. Kutrowski, T. Wojtowicz, P. Kossacki, V. Ciulin, and J. Kossut, Phys. Status Solidi B 229, 791 (2002). 